\title{
Quasi-Bi-Hamiltonian Systems Obtained from Constrained Flows
}

Yunbo ZENG

Department of Mathematical Sciences, Tsinghua University, Beijing 100084, China

E-mail: yzengtsinghua.edu.cn

\begin{abstract}
An infinite number of families of quasi-bi-Hamiltonian (QBH) systems can be constructed from the constrained flows of soliton equations. The Nijenhuis coordinates for the QBH systems are proved to be exactly the same as the separation variables introduced by the Lax matrices for the constrained flows.
\end{abstract}

\section{Introduction}

For a finite-dimensional integrable Hamiltonian system (FDIHS) with degree $2 n$, a vector field, $X$, is said to be a $\mathrm{QBH}$ vector field with respect to two compatible and nondegenerated Poisson tensors, $\theta_{0}$ and $\theta_{1}$, if there exist two integrals of motion $F_{1}, E_{1}$ and an integrating factor $\rho$, such that $[1,2]$

$$
X=\theta_{0} \nabla F_{1}=\frac{1}{\rho} \theta_{1} \nabla E_{1} .
$$

The Nijenhuis tensor $\Phi=\theta_{1} \theta_{0}^{-1}$ has $n$ distinct eigenvalues $\boldsymbol{\mu}=\left(\mu_{1}, \ldots, \mu_{n}\right)$ [3]. One can construct a canonical transformation $(\boldsymbol{q}, \boldsymbol{p}) \mapsto(\boldsymbol{\mu}, \boldsymbol{\nu})((\boldsymbol{\mu}, \boldsymbol{\nu})$ referred to as the Nijenhuis coordinates) and the FDIHS in the Nijenhuis coordinates is separable. Several QBH systems are presented and some relationship between $\mathrm{BH}$ and QBH structure is discussed in $[1,2,4,5]$. The aims of this paper is to show how to construct an infinite number of families of QBH systems from the constrained flows of soliton equations [6-10] and to prove that the Nijenhuis coordinates for the underlying families of QBH systems are exactly the same as the separated variables introduced by the Lax matrices [11-13].

\section{New QBH system}

For Jaulent-Miodek (JM) spectral problem [14]

$$
\left(\begin{array}{l}
\psi_{1} \\
\psi_{2}
\end{array}\right)_{x}=U(u, \lambda)\left(\begin{array}{l}
\psi_{1} \\
\psi_{2}
\end{array}\right), \quad U(u, \lambda)=\left(\begin{array}{cc}
0 & 1 \\
\lambda^{2}-u_{1} \lambda-u_{0} & 0
\end{array}\right), \quad u=\left(\begin{array}{l}
u_{1} \\
u_{0}
\end{array}\right)
$$

the Jaulent-Miodek hierarchy reads

$$
u_{t_{n}}=\left(\begin{array}{l}
u_{1} \\
u_{0}
\end{array}\right)_{t_{n}}=J\left(\begin{array}{l}
b_{n+2} \\
b_{n+1}
\end{array}\right)=J \frac{\delta H_{n}}{\delta u}, \quad n=1,2, \ldots
$$


where $H_{n}=\frac{1}{n}\left(2 b_{n+3}-u_{1} b_{n+2}\right)$

$$
\begin{aligned}
& b_{0}=b_{1}=0, \quad b_{2}=1, \quad\left(\begin{array}{c}
b_{k+2} \\
b_{k+1}
\end{array}\right)=L\left(\begin{array}{c}
b_{k+1} \\
b_{k}
\end{array}\right), \quad k=1,2, \ldots, \\
& L=\left(\begin{array}{cc}
u_{1}-\frac{1}{2} D^{-1} u_{1, x} & \frac{1}{4} D^{2}+u_{0}-\frac{1}{2} D^{-1} u_{0, x} \\
1 & 0
\end{array}\right), \\
& D=\frac{\partial}{\partial x}, \quad D D^{-1}=D^{-1} D=1 .
\end{aligned}
$$

Under zero boundary condition we have $\frac{\delta \lambda}{\delta u}=\left(\begin{array}{c}\lambda \psi_{1}^{2} \\ \psi_{1}^{2}\end{array}\right)$. The constrained flows of $(2.2)$ are defined by [6-10]:

$$
\begin{aligned}
& \Psi_{1, x}=\Psi_{2}, \quad \Psi_{2, x}=\Lambda^{2} \Psi_{1}-u_{1} \Lambda \Psi_{1}-u_{0} \Psi_{1}, \\
& \frac{\delta H_{l}}{\delta u}-\frac{1}{2} \sum_{j=1}^{N} \frac{\delta \lambda_{j}}{\delta u}=\left(\begin{array}{c}
b_{l+2} \\
b_{l+1}
\end{array}\right)-\frac{1}{2}\left(\begin{array}{c}
\left\langle\Lambda \Psi_{1}, \Psi_{1}\right\rangle \\
\left\langle\Psi_{1}, \Psi_{1}\right\rangle
\end{array}\right)=0 .
\end{aligned}
$$

Hereafter we denote the inner product in $\mathbf{R}^{N}$ by $\langle\cdot, \cdot\rangle$ and $\Psi_{i}=\left(\psi_{i 1}, \ldots, \psi_{i N}\right)^{T}, i=1,2$, $\Lambda=\operatorname{diag}\left(\lambda_{1}, \ldots, \lambda_{N}\right)$ for $N$ distinct $\lambda_{j}$.

The third constrained flow $(l=4)$ can be transformed into a FDIHS

$$
\begin{aligned}
P_{x}= & \theta_{0} \nabla F_{1}, \\
P= & \left(\Psi_{1}^{T}, q_{1}, q_{2}, \Psi_{2}^{T}, p_{1}, p_{2}\right)^{T}, \quad \theta_{0}=\left(\begin{array}{cc}
0 & I_{(N+2) \times(N+2)} \\
-I_{(N+2) \times(N+2)} & 0
\end{array}\right), \\
F_{1}= & \frac{1}{2}\left\langle\Psi_{2}, \Psi_{2}\right\rangle-\frac{1}{2}\left\langle\Lambda^{2} \Psi_{1}, \Psi_{1}\right\rangle+\frac{1}{2} q_{1}\left\langle\Lambda \Psi_{1}, \Psi_{1}\right\rangle+\frac{1}{2} q_{2}\left\langle\Psi_{1}, \Psi_{1}\right\rangle \\
& -8 p_{1} p_{2}+10 q_{1} p_{2}^{2}-\frac{5}{16} q_{1}^{3} q_{2}-\frac{3}{8} q_{1} q_{2}^{2}-\frac{7}{128} q_{1}^{5} .
\end{aligned}
$$

The Lax matrix $Q \equiv\left(\begin{array}{cc}A(\lambda) & B(\lambda) \\ C(\lambda) & -A(\lambda)\end{array}\right)$ for FDIHS (2.4) is given by the method $[7,8]$

$$
\begin{aligned}
A(\lambda)= & 2 p_{2} \lambda+2 p_{1}-2 q_{1} p_{2}-\frac{1}{2} \sum_{j=1}^{N} \frac{\psi_{1 j} \psi_{2 j}}{\lambda-\lambda_{j}} \\
B(\lambda)= & \lambda^{2}+\frac{1}{2} q_{1} \lambda+\frac{3}{8} q_{1}^{2}+\frac{1}{2} q_{2}+\frac{1}{2} \sum_{j=1}^{N} \frac{\psi_{1 j}^{2}}{\lambda-\lambda_{j}}, \\
C(\lambda)= & \lambda^{4}-\frac{1}{2} q_{1} \lambda^{3}-\left(\frac{1}{2} q_{2}+\frac{1}{8} q_{1}^{2}\right) \lambda^{2}+\left(\frac{1}{4} q_{1}^{3}+\frac{1}{2} q_{1} q_{2}-\frac{1}{2}\left\langle\Psi_{1}, \Psi_{1}\right\rangle\right) \lambda \\
& +\frac{1}{4} q_{2}^{2}-\frac{5}{64} q_{1}^{4}-4 p_{2}^{2}-\frac{1}{2}\left\langle\Lambda \Psi_{1}, \Psi_{1}\right\rangle+\frac{1}{2} q_{1}\left\langle\Psi_{1}, \Psi_{1}\right\rangle-\frac{1}{2} \sum_{j=1}^{N} \frac{\psi_{2 j}^{2}}{\lambda-\lambda_{j}} .
\end{aligned}
$$

We can show the following propositions. 
Proposition 1. The FDIHS (2.4) possesses the QBH representation (1.1) with

$$
\begin{aligned}
& \rho=\left.B(\lambda)\right|_{\lambda=0}=\frac{3}{8} q_{1}^{2}+\frac{1}{2} q_{2}-\frac{1}{2}\left\langle\Lambda^{-1} \Psi_{1}, \Psi_{1}\right\rangle, \\
& E_{1}=\left.\left[A^{2}(\lambda)+B(\lambda) C(\lambda)\right]\right|_{\lambda=0}, \quad \theta_{1}=\left(\begin{array}{cc}
0_{(N+2) \times(N+2)} & A_{1} \\
-A_{1}^{T} & B_{1}
\end{array}\right), \\
& A_{1}=\left(\begin{array}{ccc}
\lambda & -\frac{1}{4} \Psi_{1} & 0_{N \times 1} \\
0_{1 \times N} & q_{1} & 1 \\
2 \Psi_{1}^{T} & -\frac{1}{2} q_{2}-\frac{15}{8} q_{1}^{2} & -\frac{3}{2} q_{1}
\end{array}\right), \quad B_{1}=\left(\begin{array}{ccc}
0_{N \times N} & \frac{1}{4} \Psi_{2} & 0_{N \times 1} \\
-\frac{1}{4} \Psi_{2}^{T} & 0 & p_{2} \\
0_{1 \times N} & -p_{2} & 0
\end{array}\right) .
\end{aligned}
$$

The eigenvalues $\mu_{1}, \ldots, \mu_{N+2}$ of $\theta_{1} \theta_{0}^{-1}$ are defined by the roots of the equation $f(\lambda)=$ $\left|\lambda I-A_{1}\right|=0$ which gives rise to $[4,5]$

$$
\begin{aligned}
& \mu_{j}=f_{j}\left(\Psi_{1}, q_{1}, q_{2}\right), \quad \psi_{1 j}=g_{j}(\boldsymbol{\mu}), \quad q_{1}=g_{N+1}(\boldsymbol{\mu}), \quad q_{2}=g_{N+2}(\boldsymbol{\mu}), \\
& \nu_{j}=\frac{\partial S}{\partial \mu_{j}}=\sum_{j=1}^{N} \psi_{2 j} \frac{\partial g_{j}}{\partial \mu_{j}}+p_{1} \frac{\partial g_{N+1}}{\partial \mu_{j}}+p_{2} \frac{\partial g_{N+2}}{\partial \mu_{j}}, \quad j=1, \ldots, N+2 .
\end{aligned}
$$

It is known [11-13] that the separated variables $(\overline{\boldsymbol{\mu}}, \overline{\boldsymbol{\nu}})$ for $(2.4)$ can be constructed by means of the Lax matrix in the following way:

$$
\begin{aligned}
& B(\lambda)=\frac{R(\lambda)}{K(\lambda)}, \quad R(\lambda)=\prod_{k=1}^{N+2}\left(\lambda-\bar{\mu}_{k}\right), \quad K(\lambda)=\prod_{k=1}^{N}\left(\lambda-\lambda_{k}\right), \\
& \bar{\nu}_{k}=-A\left(\bar{\mu}_{k}\right), \quad k=1, \ldots, N+2 .
\end{aligned}
$$

Proposition 2. The Nijenhuis coordinates $(\boldsymbol{\mu}, \boldsymbol{\nu})$ defined by Eqs. (2.7) are exactly the same as the separated variables $(\overline{\boldsymbol{\mu}}, \overline{\boldsymbol{\nu}})$ defined by Eqs. (2.8).

\section{Infinite numbers of families of QBH systems}

Consider the following polynomial second order spectral problem [15]

$$
\left(\begin{array}{l}
\psi_{1} \\
\psi_{2}
\end{array}\right)_{x}=U(u, \lambda)\left(\begin{array}{l}
\psi_{1} \\
\psi_{2}
\end{array}\right), \quad U(u, \lambda)=\left(\begin{array}{cc}
0 & 1 \\
-\sum_{i=0}^{m} u_{i} \lambda^{i} & 0
\end{array}\right) .
$$

In the same way, one obtains the first constrained flows associated with spectral problem (3.1) [10]

$$
P_{x}=\theta_{0} \nabla F_{1},
$$

where

$$
\begin{aligned}
& P=\left(\Psi_{1}^{T}, \Psi_{2}^{T}\right)^{T}, \quad \theta_{0}=\left(\begin{array}{cc}
0 & I_{N \times N} \\
-I_{N \times N} & 0
\end{array}\right), \\
& F_{1}=\frac{1}{2}\left\langle\Psi_{2}, \Psi_{2}\right\rangle+\sum_{j=0}^{m}\left(-\frac{1}{2}\right)^{j+1} \sum_{l_{1}+\cdots+l_{j+1}=m-j}\left\langle\Lambda^{l_{1}} \Psi_{1}, \Psi_{1}\right\rangle \cdots\left\langle\Lambda^{l_{j+1}} \Psi_{1}, \Psi_{1}\right\rangle .
\end{aligned}
$$


Proposition 3. The first family of FDIHS (3.2) $(m=1,2, \ldots)$ possesses the $Q B H$ representation (1.1) with $\theta_{1}=\left(\begin{array}{cc}0_{N \times N} & A_{1} \\ -A_{1}^{T} & B_{1}\end{array}\right)$ and

$$
\begin{aligned}
A_{1}= & \lambda-\frac{1}{2} \Psi_{1} \Psi_{1}^{T}, \quad B_{1}=\frac{1}{2} \Psi_{2} \Psi_{1}^{T}-\frac{1}{2} \Psi_{1} \Psi_{2}^{T}, \\
\rho= & \left.B(\lambda)\right|_{\lambda=0}=1-\frac{1}{2}\left\langle\Lambda^{-1} \Psi_{1}, \Psi_{1}\right\rangle, \\
E_{1}= & \frac{1}{2}\left\langle\Lambda^{-1} \Psi_{2}, \Psi_{2}\right\rangle+\frac{1}{4}\left[\left\langle\Lambda^{-1} \Psi_{1}, \Psi_{2}\right\rangle^{2}-\left\langle\Lambda^{-1} \Psi_{1}, \Psi_{1}\right\rangle\left\langle\Lambda^{-1} \Psi_{2}, \Psi_{2}\right\rangle\right] \\
& +\sum_{j=0}^{m}\left(-\frac{1}{2}\right)^{j+1} \sum_{l_{1}+\cdots+l_{j+1}=m-j}\left\langle\Lambda^{l_{1}} \Psi_{1}, \Psi_{1}\right\rangle \cdots\left\langle\Lambda^{l_{j+1}-1} \Psi_{1}, \Psi_{1}\right\rangle .
\end{aligned}
$$

The second constrained flows associated with Eq. (3.1) is [10]

$$
P_{x}=\theta_{0} \nabla F_{1},
$$

where

$$
\begin{aligned}
P= & \left(\Psi_{1}^{T}, q, \Psi_{2}^{T}, p\right)^{T}, \quad \theta_{0}=\left(\begin{array}{cc}
0 & I_{(N+1) \times(N+1)} \\
-I_{(N+1) \times(N+1)} & 0
\end{array}\right), \\
F_{1}= & \frac{1}{2}\left\langle\Psi_{2}, \Psi_{2}\right\rangle+\left(-\frac{1}{2} q\right)^{m+2}-4 p^{2} \\
& +\sum_{i=0}^{m} q^{i} \sum_{j=1}^{\left[\frac{m+2-i}{2}\right]} \beta_{i, j} \sum_{l_{1}+\cdots+l_{j}=m+2-i-2 j}\left\langle\Lambda^{l_{1}} \Psi_{1}, \Psi_{1}\right\rangle \cdots\left\langle\Lambda^{l_{j}} \Psi_{1}, \Psi_{1}\right\rangle .
\end{aligned}
$$

Proposition 4. The second family of FDIHS (3.3) $(m=1,2, \ldots)$ possesses the $Q B H$ representation (1.1) with

$$
\begin{aligned}
\theta_{1}= & \left(\begin{array}{cc}
0_{(N+1) \times(N+1)} & A_{1} \\
-A_{1}^{T} & B_{1}
\end{array}\right), \quad A_{1}=\left(\begin{array}{cc}
\lambda & -\frac{1}{4} \Psi_{1} \\
2 \Psi_{1}^{T} & -\frac{1}{2} q
\end{array}\right), \\
B_{1}= & \left(\begin{array}{cc}
0_{N \times N} & \frac{1}{4} \Psi_{2} \\
-\frac{1}{4} \Psi_{2}^{T} & 0
\end{array}\right), \quad \rho=\left.B(\lambda)\right|_{\lambda=0}=\frac{1}{2} q-\frac{1}{2}\left\langle\Lambda^{-1} \Psi_{1}, \Psi_{1}\right\rangle, \\
E_{1}= & 2 p\left\langle\Lambda^{-1} \Psi_{1}, \Psi_{2}\right\rangle+\frac{1}{4} q\left\langle\Lambda^{-1} \Psi_{2}, \Psi_{2}\right\rangle+4 p^{2}-\left(-\frac{1}{2} q\right)^{m+2} \\
& -\sum_{i=0}^{m} q^{i} \sum_{j=1}^{\left.\frac{m+2-i}{2}\right]} \beta_{i, j} \sum_{l_{1}+\cdots+l_{j}=m+2-i-2 j}\left\langle\Lambda^{l_{1}} \Psi_{1}, \Psi_{1}\right\rangle \cdots\left\langle\Lambda^{l_{j}} \Psi_{1}, \Psi_{1}\right\rangle \\
& +\frac{1}{4}\left[\left\langle\Lambda^{-1} \Psi_{1}, \Psi_{2}\right\rangle^{2}-\left\langle\Lambda^{-1} \Psi_{1}, \Psi_{1}\right\rangle\left\langle\Lambda^{-1} \Psi_{2}, \Psi_{2}\right\rangle\right]-\frac{1}{2} \sum_{i=0}^{m+1} q^{i} \sum_{j=0}^{\left.\frac{m+1-i}{2}\right]} \beta_{i, j} \\
& \times \sum_{l_{1}+\cdots+l_{j+1}=m+1-i-2 j}\left\langle\Lambda^{l_{1}} \Psi_{1}, \Psi_{1}\right\rangle \cdots\left\langle\Lambda^{l_{j}} \Psi_{1}, \Psi_{1}\right\rangle\left\langle\Lambda^{l_{j+1}-1} \Psi_{1}, \Psi_{1}\right\rangle .
\end{aligned}
$$


The separation of variables for FDIHSs (3.2) and (3.3) was studied in [10]. We can show that

Proposition 5. The Nijenhuis coordinates $(\boldsymbol{\mu}, \boldsymbol{\nu})$ for FDIHS (3.2) and (3.3) are exactly the same as the generalized elliptic and the generalized parabolic coordinates $(\overline{\boldsymbol{\mu}}, \overline{\boldsymbol{\nu}})$ defined by Lax matrix for (3.2) and (3.3), respectively.

In general, we can obtain infinite number of families of QBH systems from the higherorder constrained flows associated with Eq. (3.1).

\section{References}

[1] Caboz R, Ravoson V and Gavrilov L, J. Phys. A, 1991, V.24, L523.

[2] Brouzet R, Caboz R, Rabenivo J and Ravoson V, J. Phys. A, 1996, V.29, 2069.

[3] Magri F and Marsico T, Electromagnetism and Geometrical Structures, Editor G Ferrarese, Bologna, Pitagora, 1996, 207.

[4] Morosi C and Tondo G, J. Phys. A, 1997, V.30, 2799.

[5] Blaszak M, J. Math. Phys., 1998, V.39, 3213.

[6] Zeng Y, Phys. Lett. A, 1991, V.160, 541.

[7] Zeng Y and Li Y, J. Phys. A, 1993, V.26, L273.

[8] Zeng Y, Physica D, 1994, V.73, 171.

[9] Ragnisco O and Rauch-Wojciechowski S, Inverse Problems, 1992, V.8, 245.

[10] Zeng Y and Lin R, J. Math. Phys., 1998, V.39, 5964.

[11] Sklyanin E K, Prog. Theor. Phys. Suppl., 1995, V.118, 35.

[12] Kuznetsov V B, J. Math. Phys., 1992, V.33, 3240.

[13] Harnad J and Winternitz P, Comm. Math. Phys., 1995, V.172, 263.

[14] Jaulent M and Miodek K, Lett. Math. Phys., 1976, V.1, 243.

[15] Antonowicz M and Fordy A P, Comm. Math. Phys., 1989, V.124, 465. 